\title{
Modeling Reflective Bistability in Vertical-Cavity Semiconductor Optical Amplifiers
}

\author{
A. Hurtado, A. Gonzalez-Marcos, and J. A. Martin-Pereda, Senior Member, IEEE
}

\begin{abstract}
The characteristics of optical bistability in a vertical-cavity semiconductor optical amplifier (VCSOA) operated in reflection are reported. The dependences of the optical bistability in VCSOAs on the initial phase detuning and on the applied bias current are analyzed. The optical bistability is also studied for different numbers of superimposed periods in the top distributed bragg reflector (DBR) that conform the internal cavity of the device. The appearance of the $X$-bistable and the clockwise bistable loops is predicted theoretically in a VCSOA operated in reflection for the first time, to the best of our knowledge. Moreover, it is also predicted that the control of the VCSOA's top reflectivity by the addition of new superimposed periods in its top DBR reduces by one order of magnitude the input power needed for the assessment of the $X$ - and the clockwise bistable loop, compared to that required in in-plane semiconductor optical amplifiers. These results, added to the ease of fabricating two-dimensional arrays of this kind of device could be useful for the development of new optical logic or optical signal regeneration devices.
\end{abstract}

Index Terms-Optical bistability (OB), optical logic, verticalcavity semiconductor optical amplifier (VCSOA).

\section{INTRODUCTION}

O PTICAL BISTABILITY (OB) in semiconductor laser amplifiers has been a very intensive field of research in the recent past (for a review, see [1]). The physical mechanism that causes the optical bistability in laser amplifiers is based on a dispersive optical nonlinearity: an optical resonator is filled with a medium whose refractive index is dependent on the incident optical power. Under external optical power injection, due to the change in the refractive index and the saturation of gain, the resonant frequency moves toward longer wavelengths depending on the amount of the injected power and, exceeding a threshold value, the optical bistability can be observed [2], [3]. The dispersive optical bistability in laser amplifiers has been studied in Fabry-Perot semiconductor optical amplifiers (FPSOAs) [1]-[9] and in distributed feedback semiconductor optical amplifiers (DFBSOAs) [10]-[12], and this kind of device has already been used for the development of optical logic elements [13], [14], optical signal regeneration [15], and optical flip-flop operations [16].

In the last years, the study of vertical-cavity semiconductor optical amplifiers (VCSOAs) has also attracted attention [17]-[20]. The VCSOA consists of a vertical resonant cavity

Manuscript received July 21, 2004; revised October 8, 2004. This work was supported in part by the MCyT under Grant TIC2003-04309.

The authors are with the Department of Photonic Technology, Universidad Politécnica de Madrid, Madrid 28040, Spain (e-mail: hurtado@tfo.upm.es; agonmar@tfo.upm.es; jamp@tfo.upm.es).

Digital Object Identifier 10.1109/JQE.2004.841500 formed by placing an active region between two distributed Bragg reflectors (DBRs). As a consequence of its particular vertical structure, the VCSOA has a very small active region and therefore the top and bottom DBRs should have high reflectivity values in order to be able to obtain a considerable amount of power at its output; also, because of the small length of the cavity, the VCSOA has single-mode operation. The use of VCSOAs adds new advantages to those exhibited by their edge-emitting counterparts. Because of its vertical structure, the fabrication costs are considerably reduced and wafer testing is allowed. Also, the VCSOAs are suitable for the production of two-dimensional (2-D) arrays for optical parallel processing and optical interconnecting applications. The circular symmetry of VCSOAs results in a very high coupling efficiency to optical fiber. Finally, the single-mode operation of VCSOAs eliminates mode-partitioning noise.

Bohn and McInerney [21] reported the observation of dispersive bistability on an optically pumped VCSOA. Sánchez et al. [22]-[24] have recently studied the nonlinear gain properties in an electrically pumped VCSOA working in reflection. They observed differential gain and optical bistability characterized by the appearance of anticlockwise bistable loops in their I/O power relations with a high ON-OFF contrast ratio and optical gain [22]-[24]. As the switching power was considerably reduced by electrical pumping, the VCSOA under this configuration has been proposed as an alternative device for all-optical signal regeneration and optical logic applications. Recently, Wen et al. [25] have reported a vertical-cavity AND gate, using the differential gain characteristic exhibited by the electrically pumped VCSOA.

Along the lines of this work, we present a theoretical study of the reflective optical bistability occurring in a VCSOA, as we report its strong dependence on the applied bias current and on the initial phase detuning. The limits in the observation of optical bistability in terms of these two parameters will also be analyzed. We predict, for the first time to the best of our knowledge, the appearance of the X-bistable and the clockwise bistable loop. Finally, as in the VCSOA, the facet reflectivities can be precisely determined by controlling in the fabrication process the number of superimposed periods compounding the DBRs, and the influence of the VCSOA bistable behavior on the top DBR period number (and, consequently, reflectivity) is also reported. It is predicted that the change in the VCSOAs structure, by incrementing the number of periods that form the top DBR (through where the light is injected and collected) modifies the bistable characteristics of the VCSOA. These changes on the VCSOA's configuration lead to the appearance of the $\mathrm{X}$-bistable and the clockwise bistable loops at an input power 
value one order of magnitude lower than that previously reported for FPSOAs [8], [9]. This theoretical prediction could open the door for new applications of this kind of device in the optical logic field. The VCSOAs could also be used as fundamental components of the Optical Logic Programmable Logic Cell (OPLC), previously reported by the authors [26]-[28].

\section{THEORY}

A commonly used approximation for the modeling of VCSOAs is the Fabry-Perot approach [17]-[20], where the top and bottom DBRs are replaced by highly reflectivity mirrors separated by an effective cavity length conforming a Fabry-Perot cavity. This effective cavity length includes the penetration of the optical field into the mirrors. The reflectivity of the top and bottom DBRs is given by [17], [29]

$$
R_{\mathrm{DBR}}=\left(\frac{1-q p^{2 m-1} a}{1+q p^{2 m-1} a}\right)^{2}
$$

where $m$ is the number of periods of the Bragg reflectors, and $p, q$, and $a$ are, respectively, the low-to-high refractive index ratio of the two DBR layers at the first and last DBR interfaces. For the assessment of bistability, the signal to be injected into the VCSOA must be slightly detuned to the long-wavelength side of the resonance wavelength of the VCSOA [22], [23]. This feature allows the wavelength dependence of the DBR reflectivity to be ignored, since the reflection bandwidth of a typical DBR is very large compared to the difference between the wavelength of the external injected signal and the resonance wavelength of the VCSOA. Also, the cavity resonance wavelength is considered equal to the DBR center wavelength [17]. As a consequence of the distributed reflectivity provided by the DBRs, the effective cavity length $L_{c}$ is larger than the distance of the cavity formed between the two DBRs $L_{i}$, and it has to include the phase penetration depths into the top $\left(L_{p t}\right)$ and the bottom $\left(L_{p b}\right)$ DBRs in the form $L_{c}=L_{p t}+L_{i}+L_{p b}$. To calculate both phase-penetration depths into the DBRs, the following expression is applied [17], [29]:

$$
L_{p}=\frac{\lambda_{c}}{4 n_{c}} \frac{q}{1-p} \frac{\left(1-a^{2} p^{2 m-1}\right)\left(1-p^{2 m}\right)}{1-q^{2} a^{2} p^{4 m-2}}
$$

where $n_{c}$ is the averaged refractive index of the cavity formed between the two highly reflective mirrors considered in the model, and $\lambda_{c}$ is the operating wavelength. Once the VCSOA can be treated as a Fabry-Perot amplifier, the study of the reflective bistability in the VCSOA is developed making use of the model proposed by Adams [4], which is one of the most accepted models for the study of optical bistability in Fabry-Perot laser amplifiers. The model works under two basic assumptions: the spontaneous emission is neglected as it is much lower than the external injected optical power, and it is assumed that the optical intensity is uniform along the length of the cavity. The model also neglects the transversal optical mode structure. Recently, Sánchez et al. [22], [23] have also used this model to study the bistability and nonlinear gain in 20 - $\mu \mathrm{m}$-diameter VCSOAs and have demonstrated excellent agreement between theory and experimental results. The
TABLE I

Lashr Parameter Dhinitions Ushid to MOIDH, THE, VCSOA [19], [22]-[24]

\begin{tabular}{lc}
\hline \multicolumn{1}{c}{ VCSOA Parameter } & Value \\
Wavelength $(\mathrm{nm}), \lambda$ & 853.33 \\
Cavity Length $(\mu \mathrm{m}), \mathrm{L}_{\mathrm{i}}$ & $3 \cdot \lambda / \mathrm{n}_{\mathrm{c}}$ \\
Cavity refractive index, $\mathrm{n}_{\mathrm{c}}$ & 3.2 \\
Top DBR period & 16 \\
Bottom DBR periods & 25 \\
DBR high/low refractive indexes, $\left(\mu_{\mathrm{h}} / \mu_{1}\right)[19]$ & $3.45 / 2.89$ \\
Substrate refractive index $\left(\mu_{\mathrm{s}}\right)$ & 3.45 \\
Active region area $\left(\mu \mathrm{m}^{2}\right)[23]$ & $\pi \cdot 10^{2}$ \\
Confinement Factor, $\Gamma$ & 0.1 \\
lnternal Quantum Efficiency, $\eta_{\mathrm{i}}$ & 1 \\
Linear Material Gain Coeff. $\left(\mathrm{cm}^{2}\right), \mathrm{a}$ & $2.7 \cdot 10^{-16}$ \\
Linewidth Enhancement Factor, $\mathrm{b}$ & 2.7 \\
Linear Recombination Coeff. $(1 / \mathrm{s}), \mathrm{A}$ & $1 \cdot 10^{8}$ \\
Bimolecular Recomb. Coeff. $\left(\mathrm{m}^{3} / \mathrm{s}\right), \mathrm{B}$ & $10^{-16}$ \\
Auger Recombination Coeff. $\left(\mathrm{m}^{6} / \mathrm{s}\right), \mathrm{C}$ & $5 \cdot 10^{-42}$ \\
Transparency Carrier Density $\left(1 / \mathrm{m}^{3}\right), \mathrm{n}_{0}$ & $1.5 \cdot 10^{24}$ \\
Fixed Internal Loss $(1 / \mathrm{m}), \alpha$ & 1,000 \\
$\mathrm{P}_{\mathrm{x}}[22]$ & 200,000 \\
$\mathrm{P}_{\mathrm{y}}[22]$ & 260,000 \\
\hline \hline
\end{tabular}

equations relating the injected, reflected, and averaged internal power are given by [2], [9], [22]

$$
\begin{aligned}
I_{\mathrm{av}} & =\frac{\left(1-R_{t}\right)\left(1+R_{b} e^{g L_{c}}\right)\left(e^{g L_{c}}-1\right)}{\left(1-\sqrt{R_{t} R_{b}} e^{g L_{c}}\right)^{2}+4 \sqrt{R_{t} R_{b}} e^{g L_{c}} \sin ^{2} \phi} \frac{P_{\mathrm{in}}}{P_{x} g L_{c}} \\
P_{R} & =\frac{\left(\sqrt{R_{t}}-\sqrt{R_{b}} e^{g L_{c}}\right)^{2}+4 \sqrt{R_{t} R_{b}} e^{g L_{c}} \sin ^{2} \phi}{\left(1-R_{t}\right)\left(1+R_{b} e^{g L_{c}}\right)\left(e^{g L_{c}}-1\right)} I_{\mathrm{av}} P_{y} g L_{c}
\end{aligned}
$$

where $R_{t}$ and $R_{b}$ correspond to the top and bottom DBR reflectivity [calculated from (1)], $L_{c}$ is the effective length of the VCSOA, obtained from (2), $g$ is the gain per unit length, $\phi$ is the single-pass phase change, and, finally, $P_{x}$ and $P_{y}$ are scaling parameters whose value has been taken from the literature [22]. These constant parameters do not have any influence on the bistable behavior of the VCSOA and are used simply to convert the results to conventional optical power units for graphical representation. In the steady state, the net gain coefficient and the relative phase change are expressed by

$$
\begin{aligned}
g L_{c} & =\frac{\Gamma g_{0} L_{c}}{1+\left(I_{\mathrm{av}} / I_{s}\right)}-\alpha_{i} L_{c} \\
\Phi & =\Phi_{0}+\frac{\Gamma g_{0} L_{c} b}{2}\left(\frac{I_{\mathrm{av}} / I_{s}}{1+I_{\mathrm{av}} / I_{s}}\right)
\end{aligned}
$$

where $\Phi_{0}$ is the initial phase detuning [3], [4], $b$ is the linewidth enhancement factor, $\alpha_{i}$ is the internal loss coefficient, $I_{s}$ is the saturation intensity, $g_{0}$ is the unsaturated gain coefficient expressed by $g_{0}=a\left(n-n_{0}\right)$ where $a$ is the linear material gain coefficient, $n_{0}$ is the transparency carrier density, $n$ is the carrier density without external optical injection, and the confinement factor $\Gamma$ relates the volumes of the active region and the effective cavity $\Gamma=L_{a} / L_{c}$.

The temperature is considered to be kept constant in every moment by the use of temperature-controlling devices in the laboratory as in previously reported works on dispersive optical bistability occurring in VCSOAs [22]-[25]. This is why thermal effects have not been taken in consideration in the calculations. 


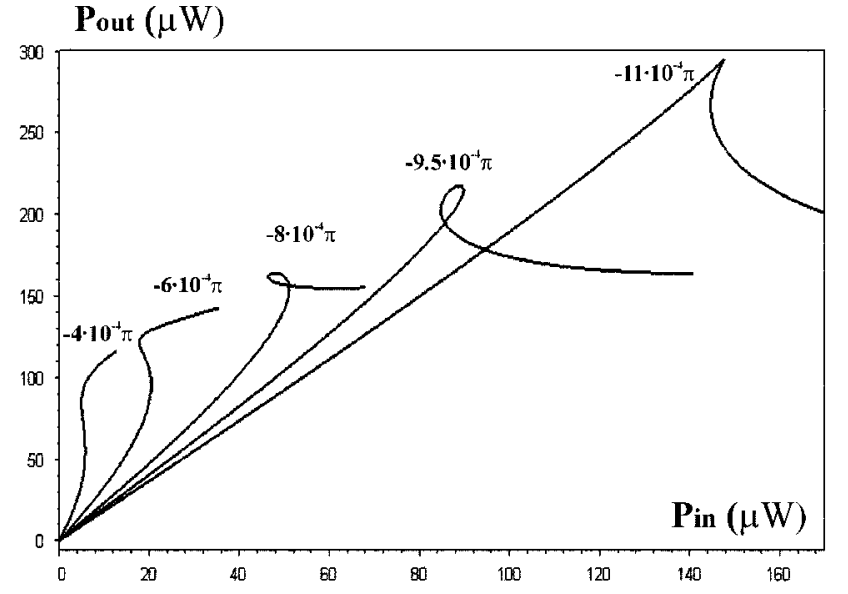

(a)

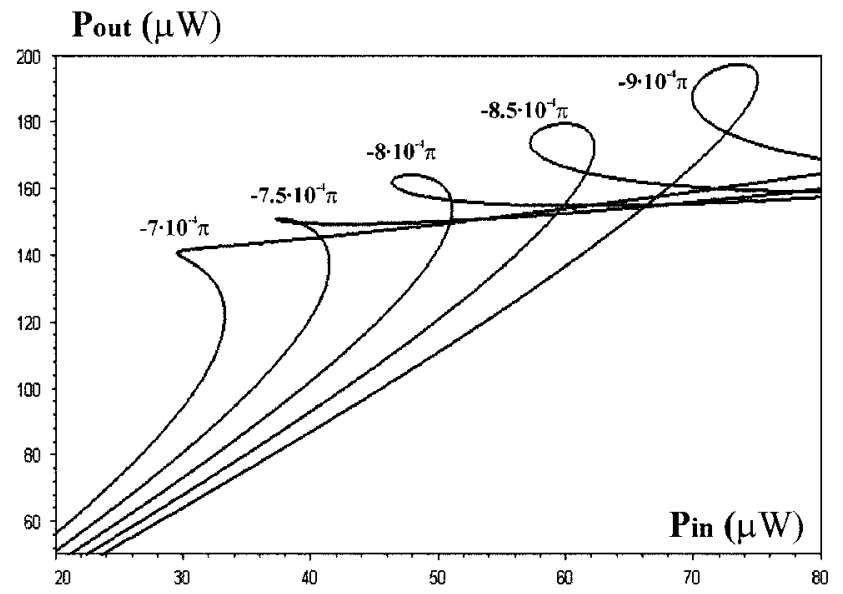

(c)

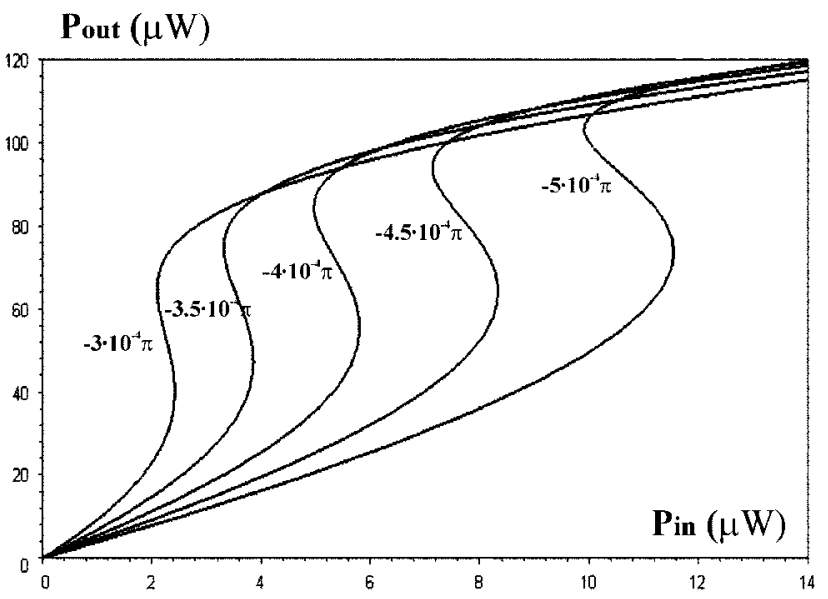

(b)

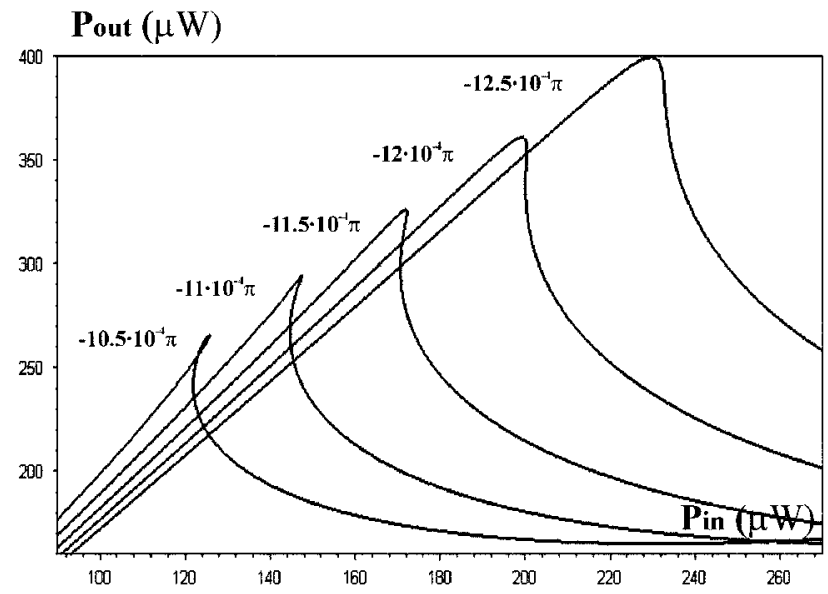

(d)

Fig. 1. Reflected versus input power, with fixed bias current equal to $99 \%$ of the threshold value for different detuning. (a) Long detuning range, from $-4 \times 10^{-4} \pi$ to $-11 \times 10^{-1} \pi$. (b) From $-3 \times 10^{-1} \pi$ to $-5 \times 10^{-1} \pi$. (c) From $-7 \times 10^{-1} \pi$ to $-9 \times 10^{-1} \pi$. (d) From $-10.5 \times 10^{-1} \pi$ to $-12.5 \times 10^{-1} \pi$.

If the temperature is not controlled, thermal effects should be considered, as it has been demonstrated that heat generation at threshold due to thermal effects can be very high [30] which may lead to the breakdown of bistability [31], [32].

\section{MODELED VCSOA}

The parameters used for the modeled VCSOA are listed in Table I. Making use of (1) and the refractive indexes values given in Table I, the reflectivity of the top and bottom DBRs are equal to $R_{t}=0.9955$ and $R_{b}=0.9993$, respectively. Applying (2), the top and bottom penetrations depths are, respectively: $L_{p t}=0.3794 \mu \mathrm{m}$ and $L_{p b}=0.3808 \mu \mathrm{m}$ that, together with the length of the cavity existing between the DBRs, equals three times the internal signal wavelength $(0.8 \mu \mathrm{m})$, leading to an effective cavity length of $L_{c}=1.56 \mu \mathrm{m}$. The approximation carried out taking account the effective cavity length and the highly reflective mirrors leads to a Fabry-Perot model of the VCSOA, and therefore a threshold condition can be applied, giving an expression for the threshold gain [33] of

$$
g_{\mathrm{th}}=\frac{1}{\Gamma L_{c}} \ln \left(\frac{1}{\sqrt{R_{t} R_{b}}}\right)+\frac{\alpha_{i}}{\Gamma}
$$

and, as the threshold gain is also a function of the carrier density at threshold in the form of $g_{\mathrm{th}}=a\left(n_{\mathrm{th}}-n_{0}\right)$, where $n_{\mathrm{th}}$ is the carrier density at threshold, the threshold current for the VCSOA is given by [33]

$$
I_{\mathrm{th}_{\mathrm{L}}}=q\left(\frac{n_{\mathrm{th}}}{\eta_{i}}\right)\left(\frac{\Gamma L_{c} \text { Area }}{\tau}\right)
$$

where $q$ is the electron charge, $\eta_{i}$ is the internal quantum efficiency, and $\tau$ is the recombination time. In this case, having substituted into (7) and (8) the parameters of the modeled device listed in Table I, the VCSOA has a calculated threshold current of $7.33 \mathrm{~mA}$.

\section{RESULTS}

Traditionally, the study of the dispersive optical bistability in semiconductor laser amplifiers has been carried out taking into account two fundamental parameters: the bias current applied to the device and the detuning existing between the resonant frequency mode of the laser amplifier and the frequency of the injected signal [6]. The influence of these parameters has also been studied in this work for the VCSOA case. Apart from the bias current and the detuning, now there is a third parameter that has a remarkable influence on the behavior of the optical reflective bistability in a VCSOA. This third parameter is the top DBR period number and its influence on the VCSOA's bistable behavior has also been reported in this work. 


\section{A. Influence of the Initial Phase Detuning}

The optical bistability occurs for negative values of the initial phase detuning as a consequence of the negative proportionality of the refractive index on the injected carrier density [3]. The external injection of light into the VCSOA results on a decrement of the carrier density. This will cause an increase of the refractive index in the active layer, which lowers the resonant cavity frequency of the VCSOA (increments the resonant cavity wavelength). The optical bistability appears when the frequency of the injected signal is lower than the resonant cavity frequency of the VCSOA. In other words, the wavelength of the injected signal must be detuned to the long-wavelength side of the VCSOA's resonant cavity wavelength for the assessment of optical bistability. Fig. 1(a)-(d) shows the bistable behavior in reflection of the modeled VCSOA at a fixed level of bias current, equal to $99 \%$ of its threshold value, and for different values of initial phase detuning. Fig. 1(a) shows the calculated reflected versus incident power in a large range of detuning values, from $-3 \times 10^{-4} \pi$ to $-11 \times 10^{-4} \pi$, where $\pi$ represents the separation between adjacent resonant modes of the VCSOA. This graphical representation predicts clearly the appearance of three different forms of bistability: the anticlock wise, the X-shaped or butterfly, and the clockwise bistable loop. Until now, in the study of the dispersive reflective bistability on VCSOAs, only the anticlockwise bistable loop had been reported [21]-[23]. This is the first time, to the best of our knowledge, that the other forms of bistability, the clockwise and the $\mathrm{X}$ - bistable loop, which have already been predicted and studied in FPSOAs and in DFBSOAs [7]-[9], are also predicted theoretically for the VCSOA case. For a constant value of bias current and small values of detuning, typical anticlockwise bistable transitions can be observed at very small values of incident power, as has already been demonstrated [22], [23]. For higher values of detuning, higher values of incident power are needed to achieve bistability, and the VCSOA shows a clockwise bistable loop in its transfer function. Finally, for intermediate values of detuning, the $\mathrm{X}$ - or butterfly bistable loop appears. Pakdeevanich et al. [9] already studied this transition from the anticlockwise to the clockwise bistability for Fabry-Perot laser amplifiers basing their results on the relationship between the input, reflected, transmitted, and averaged internal intensities

$$
\left(I_{\mathrm{ref}} / I_{s}\right)=\left(I_{\mathrm{in}} / I_{s}\right)+g L\left(I_{\mathrm{av}} / I_{s}\right)-\left(I_{\text {trans }} / I_{s}\right) .
$$

Fig. 2(a) shows the evolution of the gain per length product versus the normalized input intensity. It can be seen that, due to the decrease of the carrier concentration with increasing input intensity, the gain decays and the abrupt drop of gain is associated with the bistable transitions. On the other hand, in Fig. 2(b), the terms appearing in the right-hand side of (9) are represented as a function of the normalized input intensity. In all cases, the normalized transmitted intensity shows anticlockwise bistable loops. At the same time, and as a consequence of the evolution of the gain per length product as the initial phase detuning is increased [shown in Fig. 2(a)], the term involving the averaged intensity within the cavity shows a progressive decay in amplitude and an evolution in its bistable loop from an anticlockwise to a an X-or butterfly shape. Hence, increments in the initial
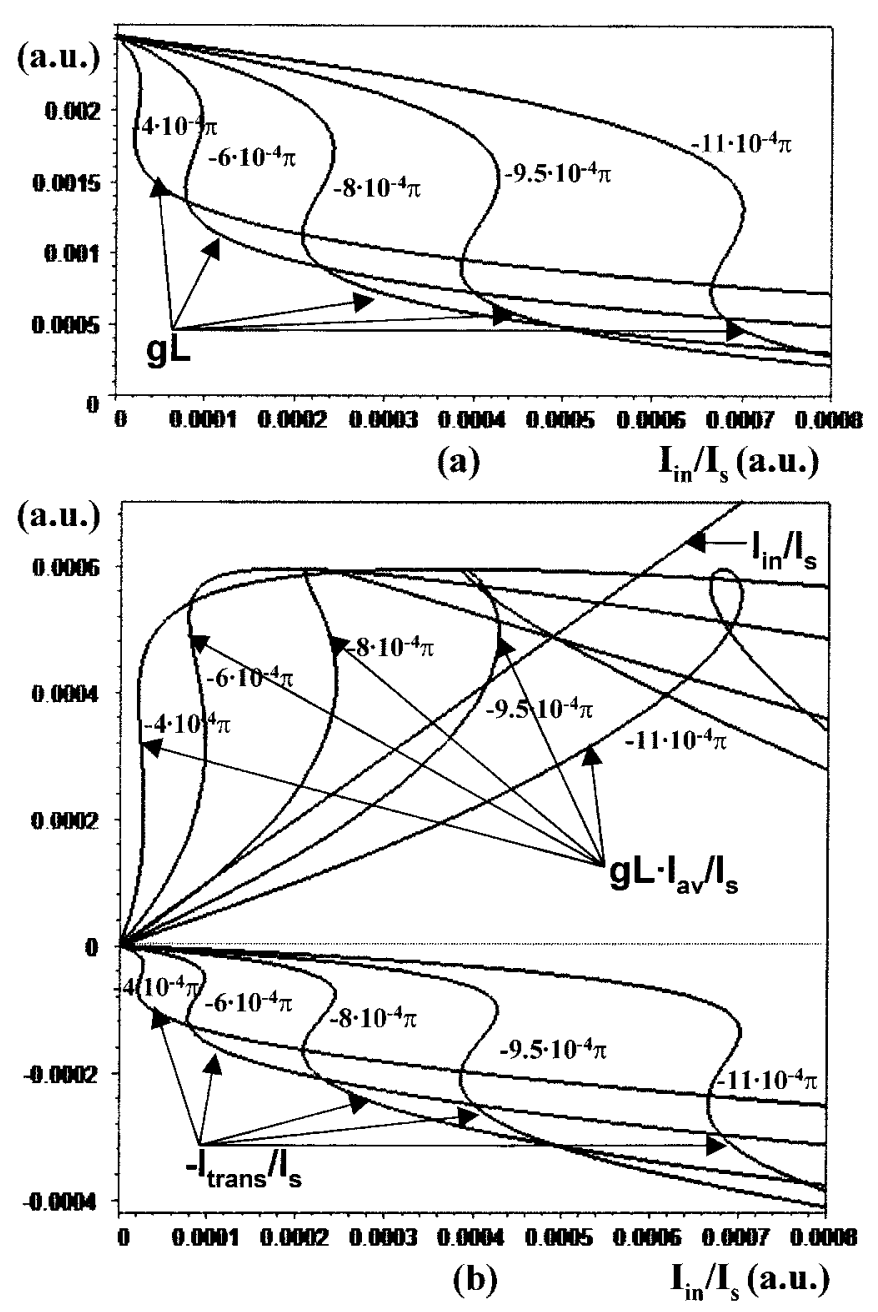

Fig. 2, (a) Gain per length product. (b) Terms on the right-hand side of (9) versus the normalized input intensity. Both graphics are at a fixed bias current of $0.99 \cdot I_{\mathrm{th}}$ and different values of detuning as indicated.

phase detuning modify the balance between the input, the transmitted, the reflected, and the averaged intensities shown in (9), leading to the assessment of the three different types of bistable loops in the reflected transfer function of the VCSOA.

Fig. 1(b)-(d) shows in detail, for different detuning ranges, the influence of the initial phase detuning in the VCSOA's reflective bistability. Apart from the change of the bistability shape as the detuning increases, for higher values of detuning, the observed bistable loops become wider and the amount of incident power needed to achieve bistablility increases from values of less than $2 \mu \mathrm{W}$, enough for the assessment of an anticlockwise bistable loop, to levels of input power above $100 \mu \mathrm{W}$, which is necessary to obtain a clockwise bistable transition. Finally, Fig. 1(d) shows, for the fixed value of bias current applied in this case, that for detuning exceeding a particular value (approximately $-12.5 \times 10^{-4} \pi$ ) the optical bistability can no longer be observed. This frequency limitation appears due to the small size of the active region of the VCSOA, which makes the gain saturate very quickly, reducing the bandwidth where the optical bistability occurs to an interval of initial phase detuning of $-12.5 \times 10^{-4} \pi$ in the longer wavelength side of the VCSOA's resonant mode. 


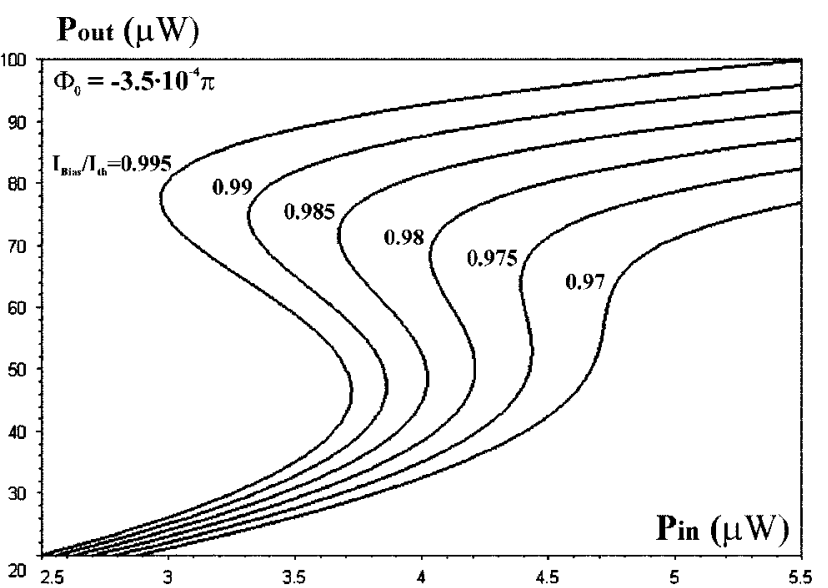

(a)

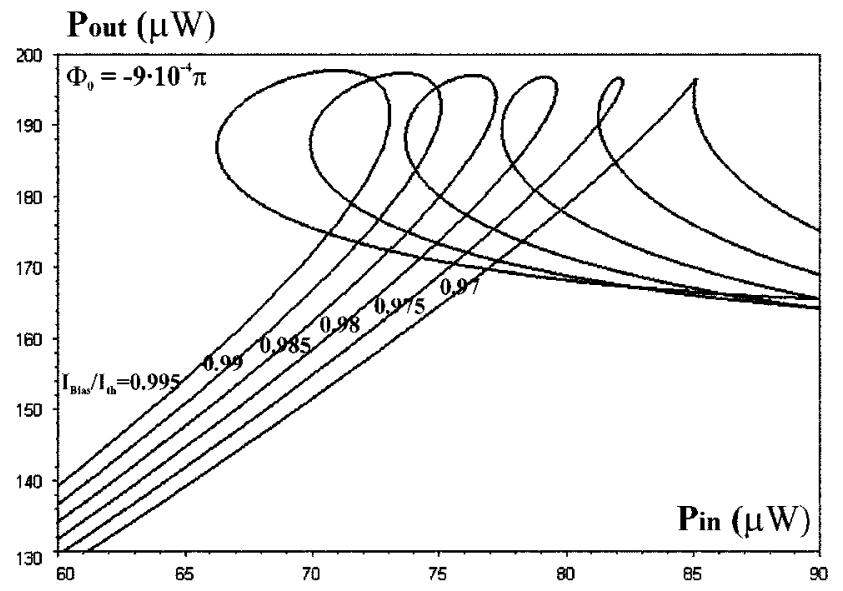

(c)

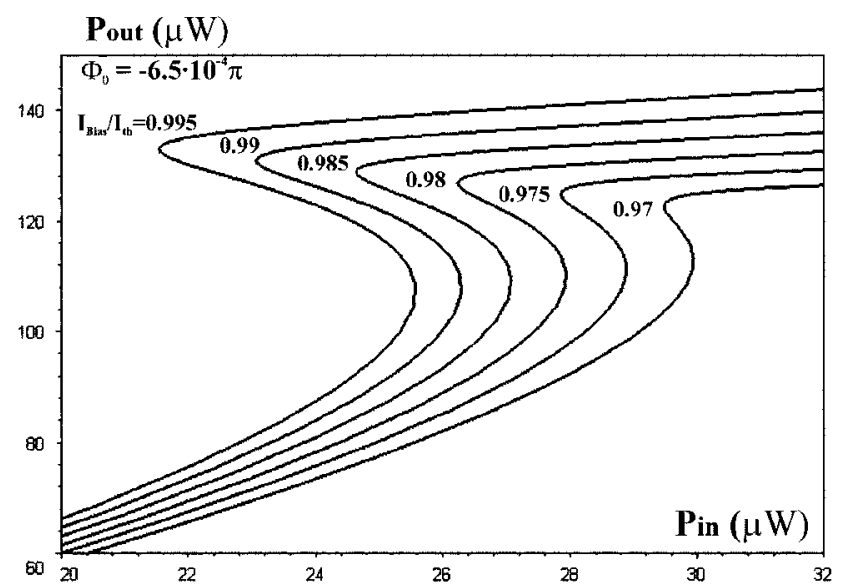

(b)

Pout $(\mu \mathrm{W})$

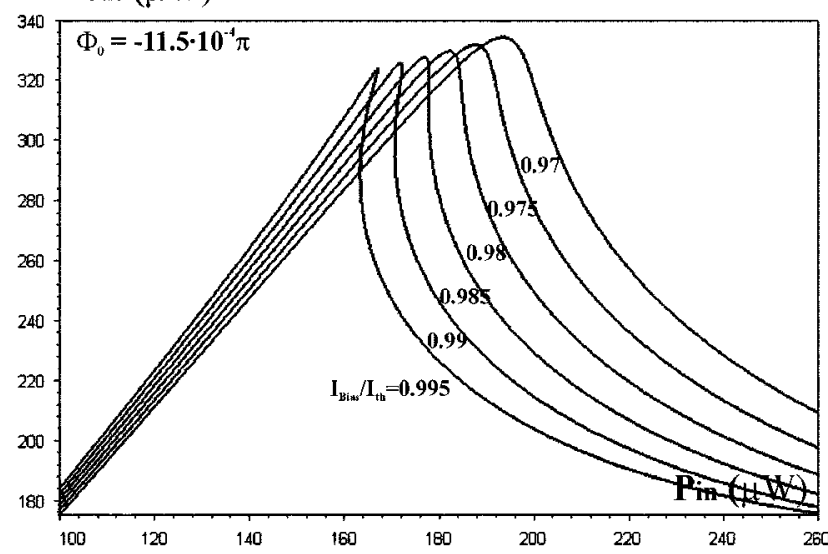

(d)

Fig. 3. Reflected versus input power at different bias currents from $99.5 \%$ to $97 \%$ of the threshold value for four constant initial phase detuning values of (a) $-3.5 \times 10^{-4} \pi$, (b) $-6.5 \times 10^{-4} \pi$, (c) $-9 \times 10^{-4} \pi$, and (d) $-11.5 \times 10^{-4} \pi$.

\section{B. Influence of the Bias Current}

The influence that the bias current applied to the VCSOA has on the reflective optical bistability has also been studied. Fig. 3(a)-(d) shows the calculated reflected versus incident power keeping constant the initial phase detuning and varying the bias current from $99.5 \%$ to $97 \%$ of the threshold value. Fig. 3(a)-(d) shows calculated results for four different values of detuning, $\left(-3.5 \times 10^{-4} \pi,-6.5 \times 10^{-4} \pi,-9 \times 10^{-4} \pi\right.$, and $\left.-11.5 \times 10^{-4} \pi\right)$ in order to represent graphically the influence of the bias current for the three different forms of bistability. In all cases, higher values of bias current result in lower power requirements to achieve bistability, wider bistable loops, and higher ON-OFF contrast ratio between output states. This evolution of the reflective bistability on the VCSOA with the applied bias current shows excellent agreement with that observed in in-plane laser amplifiers [7]-[9]. However, while for Fabry-Perot or DFB laser amplifiers the optical bistability is also observable for low values of applied bias currents, for about $70 \%$ of the threshold value or less, in the VCSOA case, this affirmation can no longer be kept. In this case, for the modeled VCSOA, the observation of optical bistability is restricted to values of bias current very near to threshold. Concretely in this case, it has been observed that the applied bias current should be higher than $96.5 \%$ of the threshold value in order to obtain optical bistability. Below this value, the optical bistability is unobservable, appearing now in the reflected versus incident power relation differential gain, as has been demonstrated in previous work [23]. Once again, the explanation for these results relies on the small size of the VCSOA's active region compared to their in-plane counterparts, which causes a very fast saturation of gain, reducing the assessment of bistability to a small range of bias currents very near to threshold.

\section{Influence of the Top DBR Period Number}

In the particular case of the VCSOA, and as it can be clearly seen from (1), the value of the reflectivity provided by the top DBR, which acts as the interface for the input and the reflected output entering and going out from the VCSOA, depends directly on the number of periods that compound it. Higher reflectivity values are obtained as the number of periods composing the top DBR is increased. Consequently, by controlling in the fabrication process the number of superimposed periods on the top DBR, its reflectivity can be precisely determined. This feature could be an additional advantage, as it may provide more flexibility in the design and fabrication of VCSOAs compared to their edge-emitting counterparts, where the facet reflectivities cannot be controlled as precisely. Equations (7) and (8) express that the threshold current of the VCSOA depend on the top and 
TABLE II

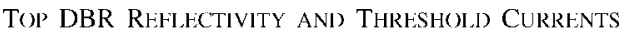
FOR DIFFERENT TOP DBR PHRIODS

\begin{tabular}{ccc}
\hline \hline $\begin{array}{c}\text { Top DBR } \\
\text { Reflectivity, Rt }\end{array}$ & $\begin{array}{c}\text { Top DBR } \\
\text { periods }\end{array}$ & $\begin{array}{c}\text { VCSOA threshold } \\
\text { current }(\mathbf{m A} \mathbf{)}\end{array}$ \\
0.9955 & 16 & 7.33 \\
0.9968 & 17 & 6.51 \\
0.9978 & 18 & 5.97 \\
0.9984 & 19 & 5.60 \\
0.9989 & 20 & 5.35 \\
0.9992 & 21 & 5.18 \\
0.9994 & 22 & 5.06 \\
\hline
\end{tabular}

bottom DBR reflectivities, so, if the number of periods of the top DBR is modified, the threshold current varies. In Table II, the values of the top DBR reflectivity and threshold current of the modeled VCSOA for different top DBR period numbers are listed.

The bottom DBR consists of 25 periods, leading to a total reflectivity of 0.9993, which is kept constant in this study. Fig. 4(a) represents five different top DBR period numbers, the reflected power versus input power at fixed values of the detuning, $-4 \times$ $10^{-4} \pi$, and applied bias current of $99 \%$ of the threshold value. For the same working conditions, in terms of bias current and detuning, the resulting behavior for each VCSOA is completely different. Once again, as in the study of the influence of the initial phase detuning, an evolution from an anticlockwise to a clockwise bistable loop with the intermediate $\mathrm{X}$ - bistable loop is appreciated. More information about the different bistable loop shapes can be extracted from the reflective spectral responses calculated for the five different cases of top DBR period numbers at fixed values of bias current and input power, which are represented graphically in Fig. 4(b). This figure shows clearly the different effects associated with the different bistable loop types.

Some important conclusions can be extracted from the graphical representation of Fig. 4(a). As the top DBR period number, and then its reflectivity, is increased, a higher input power is needed in order to achieve bistability. This would happen as a consequence of the decrement on the amount of power entering the VCSOA internal cavity, imposed by the increment on the top DBR reflectivity. Also, a change in shape is found in the bistable loop as the period number of the top DBR is increased. Once again, the explanation for these results relies on the delicate balance existing between the reflected, transmitted, and averaged internal power, expressed in (9). Fig. 5(a) shows the evolution of the gain per length product versus the normalized input intensity for the five different numbers of superimposed periods on the top DBR of the VCSOA. The gain decreases as the input power is increased, and the abrupt drop of gain occurs at the bistable switching points as a consequence of the reduction of the carrier concentration as the input power increases. However, the initial values of the gain per length product are not equal for all of the graphical representations as in Fig. 2(a). This is a consequence of the reduction of the threshold current of the VCSOA as the reflectivity of the top DBR is increased (as seen in Table II), which reduces the carrier concentration existing in the absence of external optical injection and, therefore, lowers the unsaturated gain coefficient $g_{0}$.

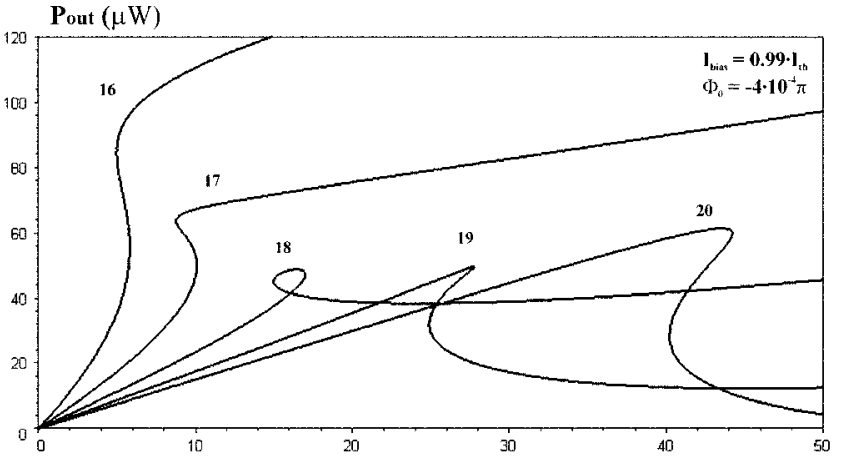

(a)

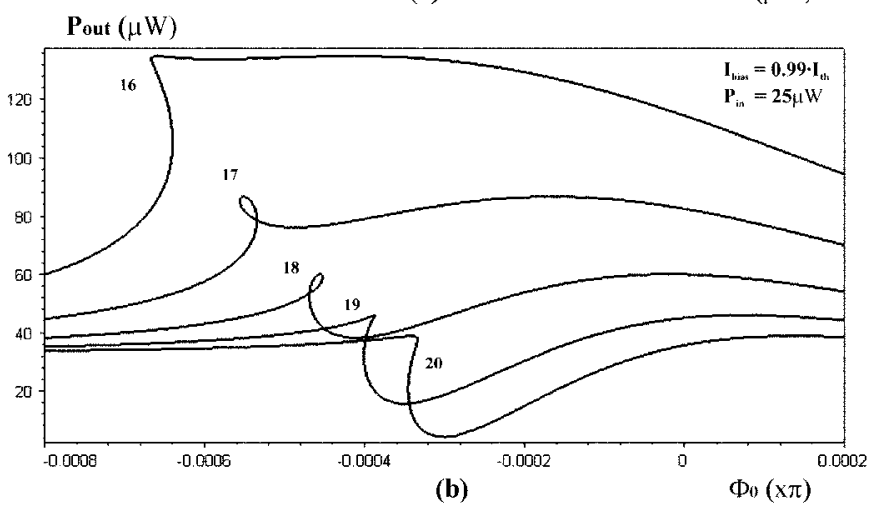

Fig. 4. (a) Calculated reflected versus input power for different top DBR period numbers at a fixed bias current of $99 \%$ of threshold and detuning of $-4 \times 10^{-4} \pi$. (b) Calculated reflected versus initial frequency detuning for the different top DBR period numbers at a fixed bias current of $0.99 \cdot I_{\mathrm{t} \mathrm{l}_{1}}$ and input power of $25 \mu \mathrm{W}$.

Fig. 5(b) represents graphically the terms appearing on the right-hand side of (9) for the same working conditions as in Fig. 5(a). Once again, the transmitted intensity shows anticlockwise bistable loops in all cases, and the term involving the gain and the average internal intensity within the cavity shows different forms of bistability, changing from anticlockwise to clockwise as the number of periods that form the top DBR is increased. This evolution of the shape of the bistability will be a consequence of two factors: the decay of the gain per length product with increasing injected power and the reduction of the initial gain per length product as the number of the periods on the top DBR is incremented. Hence, the balance between the input, transmitted, reflected, and averaged intensity within the cavity [expressed in (9)] will be modified when the number of superimposed periods on the top DBR (and, consequently, its reflectivity) is increased. This fact leads to the occurrence of the three different shapes of bistability in the input/reflected power characteristic, shown in Fig. 4(a).

It is remarkable that the control on the top DBR reflectivity reduces by more than one order of magnitude the input power requirements for the assessment of the $\mathrm{X}$ - and the clockwise bistable loop from those levels obtained for VCSOAs with "low" top DBR reflectivity [see Fig. 1(a)] and for Fabry-Perot semiconductor laser amplifiers [8], [9]. This reduced input power needed for the observation of the clockwise bistable loop, added to the acceptable contrast ratio between output states observed in Fig. 4(a), may suggest new applications of this kind of device for the development of optical logic 


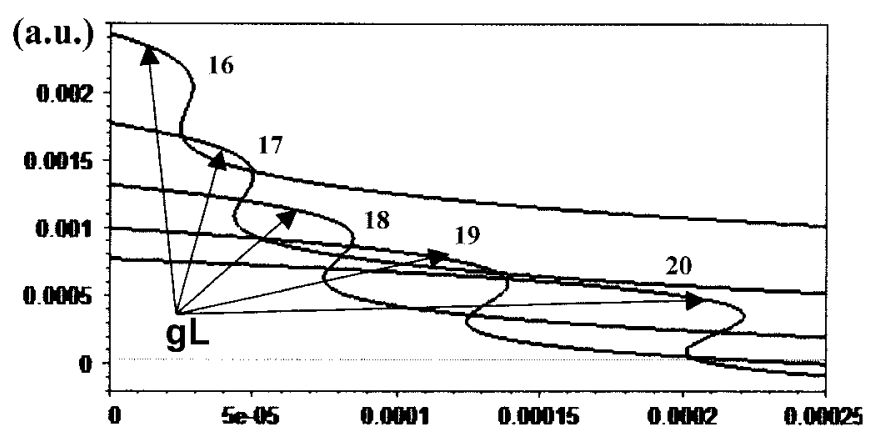

(a)

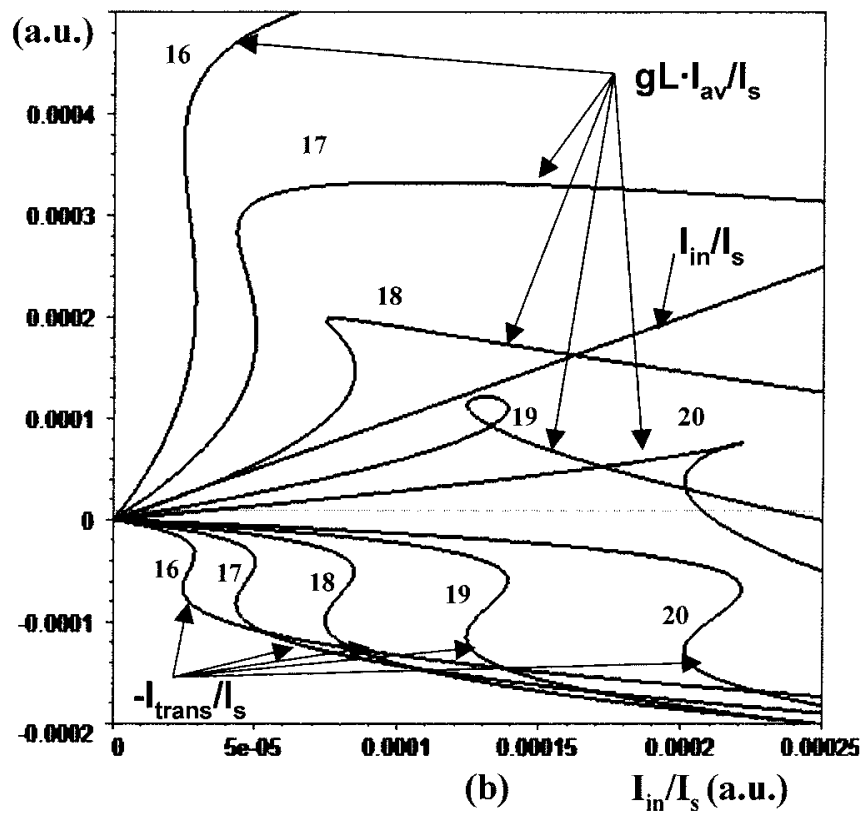

Fig. 5. (a) Gain per length product and (b) terms on the right-hand side of (9) versus the normalized input intensity. Both figures at a fixed bias current of $0.99 \cdot I_{\mathrm{th}}$ and initial phase detuning of $-4 \times 10^{-1} \pi$ and for different numbers of periods on the top DBR as indicated.

elements, including optical NAND or NOR optical gates [34], optical memory, or optical signal regeneration applications in optical communications systems. Moreover, as Wen et al. [25] report in their description of a vertical-cavity AND gate, another additional advantage associated with the use of VCSOAs is the high-frequency performance. Westlake et al. [5] demonstrated that the carrier recombination time limits the high-frequency response of $\mathrm{OB}$ in semiconductor laser amplifiers. Wen et al. [25] suggest that the VCSOA should have a better high-frequency response due to its quantum-well structure and small volume of gain medium. Finally, the ease of fabricating 2-D arrays of these devices makes the VCSOA very attractive for applications in optical parallel signal processing and optical interconnect.

\section{CONCLUSION}

The optical bistability in VCSOAs has been studied for the reflective mode of operation. The dependence of the reflective bistability in a VCSOA on the initial phase detuning and bias current is reported. It has been predicted too, for the first time, the appearance of the X-shaped or butterfly and clockwise bistable loops, as well as the presence of the usual anticlockwise bistable loop. Due to the small size of the active region, the gain saturates too quickly, leading to the existence of restrictions in the applied bias current and in the detuning for the observation of the optical bistability. In the modeled VCSOA used in this work, for the appearance of the bistability, the applied bias should be higher than the $96.5 \%$ of the threshold value, and the external injected signal frequency is restricted to a range equal to $-12.5 \times 10^{-4} \pi$ (where $\pi$ represents the spacing between adjacent resonant modes) in the longer wavelength side of the VCSOA's operating wavelength. Finally, the reflective bistability has been studied for different numbers of periods in the top DBR of the VCSOA. It has been predicted that the shape of the observed bistable loops changes from an anticlockwise to a clockwise loop as the number of periods of the top DBR, and consequently its reflectivity, is increased. In this case, the predicted input power needed for the appearance of the Xand the clockwise bistable loops are one order of magnitude lower than that reported previously for FPSOAs. This result, added to the high-frequency response of the optical bistability in VCSOAs and the ease of fabricating 2-D arrays of these devices, makes the VCSOA very attractive for the development of optical logic devices, including NAND and NOR logic gates for optical computing applications, optical memories, optical signal regeneration elements in optical communication systems, parallel optical signal processing elements, and optical interconnecting devices.

\section{REFERENCES}

[1] H. Kawaguchi, Bistabilities and Nonlinearities in Laser Diodes. Norwood, MA: Artech House, 1994.

[2] K. Otsuka and H. Iwamura, "Analysis of a multistable semiconductor light amplifier," IEEE J. Quantum Electron., vol. QE-19, no. 7, pp. 1184-1186, Jul. 1983.

[3] K. Otsuka and S. Kobayashi, "Optical bistability and nonlinear resonance in a resonant-type semiconductor laser amplifier," Electron. Lett., vol. 19, no. 7, pp. 262-263, Mar. 1983.

[4] M. J. Adams, H. J. Wetlake, M. J. O'Mahony, and I. D. Henning, "A comparison of active and passive optical bistability in semiconductors," IEEE J. Quantum Electron., vol. QE-21, no. 9, pp. 1498-1504, Sep. 1985.

[5] H. J. Westlake, M. J. Adams, and M. J. O'Mahony, "Assesment of switching speed of optical bistability in semiconductor laser amplifiers," Electron. Lett., vol. 22, no. 10, pp. 541-543, May 1986.

[6] Z. Pan, H. Lin, and M. Dagenais, "Switching power dependence on detuning and current in bistable diode laser amplifiers," Appl. Phys. Lett., vol. 58, no. 7, pp. 687-689, Feb. 1991.

[7] M. J. Adams, "Optical amplifier bistability on reflection," Opt. Quantum Electron., vol. 19, pp. S37-S45, 1987

[8] N. F. Mitchell, J. O'Gorman, J. Hegarty, and J. C. Connolly, "Optical bistability in assymetric Fabry-Perot laser-diode amplifiers," Opt. Lett., vol. 19, no. 4, pp. 269-271, Feb. 1994.

[9] P. Pakdeevanich and M. J. Adams, "Measurements and modeling of reflective bistability in 1.55- $/ \mathrm{m}$ laser diode amplifiers," IEEE J. Quantum Electron., vol. 35, no. 12, pp. 1894-1903, Dec. 1999.

[10] H. Kawaguchi, K. Inoue, T. Matsuoka, and K. Otsuka, "Bistable output characteristics in semiconductor laser injection locking," IEEE J. Quantum Electron., vol. QE-21, no. 9, pp. 1314-1317, Sep. 1985.

[11] M. J. Adams and R. Wyatt, "Optical bistability in distributed feedback semiconductor laser amplifiers," in Proc. Inst. Elect. Eng., vol. 134, Feb. 1987 , pp. 58-63.

[12] D. N. Maywar and G. P. Agrawal, "Transfer-matrix analysis of optical bistability in DFB semiconductor laser amplifiers with nonuniform gratings," IEEE J. Quantum Electron., vol. 33, no. 11, pp. 2029-2037, Nov. 1997. 
[13] W. F. Sharfin and M. Dagenais, "High contrast, $1.3 \mu \mathrm{m}$ optical AND gate with gain," Appl. Phys. Lett., vol. 48, no. 22, pp. 1510-1512, June 1986.

[14] A. Hurtado-Villavieja and A. Gonzalez-Marcos, "An all-optical programmable logic gate with $1550 \mathrm{~nm}$ laser diode amplifiers," in Proc. Opt. Computing, Engelberg, Switzerland, Apr. 21-23, 2004, pp. 5-6.

[15] K. Nonaka, Y. Noguchi, H. Tsuda, and T. Kurokawa, "Digital signal regeneration with side-injection-light-controlled bistable laser diode as a wavelength converter," IEEE Photon. Technol. Lett., vol. 7, no. 1, pp. 139-141, Jan. 1995.

[16] K. Inoue, "All-optical flip-flop operation in an optical bistable device using two lights of different frequencies," Opt. Lett., vol. 12, pp. 918-920, 1987

[17] C. Tombling, T. Saitoh, and T. Mukai, "Performance predictions for vertical-cavity semiconductor laser amplifiers," IEEE J. Quantum Electron., vol. 30, no. 11, pp. 2491-2499, Nov. 1994

[18] P. Royo, R. Koda, and L. A. Coldren, "Vertical cavity semiconductor optical amplifiers: Comparison of fabry-perot and rate equation approaches," IEEE J. Quantum Electron., vol. 38, no. 3, pp. 279-284, Mar. 2002.

[19] J. Piprek, S. Björling, and J. E. Bowers, "Design and analysis of verticalcavity semiconductor optical amplifiers," IEEE J. Quantum Electron. vol. 37, no. 1, pp. 127-134, Jan. 2001.

[20] E. S. Björling, T. Kimura, and J. E. Bowers, "Carrier-confined verticalcavity semiconductor optical amplifiers for higher gain and efficiency," IEEE J. Select. Topics Quantum Electron., vol. 9, no. 5, pp. 1374-1385, Sep./Oct. 2003.

[21] M. J. Bohn and J. G. McInerney, "Bistable output of an optically pumped vertical-cavity surface-emitting laser," J. Opt. Soc. Amer. B, vol. 14, no. 12, pp. 3430-3436, Dec. 1997.

[22] P. Wen, M. Sánchez, M. Gross, and S. Esener, "Observation of bistability in a vertical-cavity semiconductor optical amplifier (VCSOA)," Opt. Express, vol. 10 , no. 22 , pp. $1273-1278$, Oct. 2002

[23] M. Sánchez, P. Wen, M. Gross, and S. Esener, "Nonlinear gain in vertical-cavity semiconductor optical amplifiers," IEEE Photon. Technol. Lett., vol. 15, no. 4, pp. 507-509, Apr. 2003.

[24] M. D. Sánchez, P. Wen, M. Gross, and S. C. Esener, "Rate equations for modeling dispersive nonlinearity in Fabry-Perot semiconductor optical amplifiers," Opt. Express, vol. 11, no. 21, pp. 2689-2696, Oct. 2003.

[25] P. Wen, M. D. Sánchez, M. Gross, and S. Esener, "Vertical-cavity optical AND gate," Opt. Commun., vol. 219, pp. 383-387, 2003.

[26] A. González-Marcos and J. A. Martín-Pereda, "Digital chaotic output from an optically-processing element," Opt. Eng., vol. 35, pp. 525-535, 1996.

[27] - "Analysis of irregular behavior on an optical computing logic cell," Opt. Laser Technol., vol. 32, no. 6, pp. 457-466, 2000.

[28] —, "Method to analyze the influence of hysteresis in optical arithmetic units," Opt. Eng., vol. 40, pp. 2371-2385, 2001.

[29] D. I. Babic and S. W Corzine, "Analytic expressions for the reflection delay, penetration depth, and absorptance of quarter-wave dielectric mirrors," IEEE J. Quantum Electron., vol. 28, no. 2, pp. 514-524, Feb. 1992.
[30] S. F. Yu, "Theoretical analysis of polarization bistability in vertical cavity surface emitting semiconductor lasers," J. Lightw. Technol., vol. 15, no. 6, pp. 1032-1041, Jun. 1997.

[31] F. Marino, G. Catalan, P. Sanchez, S. Ball, and O. Piro, "Thermo-optical "canard orbits" and excitable limit cycles," Phys. Rev. Lett., vol. 92, no. 7, pp. $0739011(1)-0739011(4)$, Feb. 2004.

[32] S. Barland, O. Piro, M. Giudici, J. R. Tredicce, and S. Balle, "Experimental evidence of van del Pol-Fitzhugh-Nagumo dynamics in semiconductor optical amplifiers," Phys. Rev. E, vol. 68, pp. 036 209(1)-036209(6), Sep. 2003.

[33] G. P. Agrawal, Semiconductor Lasers, Past, Present, and Future. Woodbury, NY: AIP Press, 1995, pp. 145-180.

[34] J. E. Midwinter, "Light' electronics, myth or reality?", in Proc. Inst. Elect. Eng., vol. 132, Dec. 1985 , pp. $371-383$.

A. Hurtado graduated from the E.T.S. Ingenieros de Telecomunicación, Universidad Politécnica de Madrid, Madrid, Spain, in 2001, where he is currently working toward the Ph.D. degree.

His research involves optical bistability, semiconductor laser amplifiers, optical logic, and optical computing.

A. Gonzalez-Marcos received the Ph.D. degree from E.T.S. Ingenieros de Telecomunicación, Universidad Politécnica de Madrid (UPM), Madrid, Spain, in 1993.

She was previously with private companies, where she was involved in CAD-CAE topics. She was an Assistant Professor with the Universidad de Alcalá de Henares, Madrid, and an Associate Professor with the Universidad Alfonso X, Madrid. Since 1996, she has been a Professor with the E.T.S. Ingenieros de Telecomunicación, UPM. Her present interests are optical computing, the applications of chaos theory to optical communications, and the modeling of mammalian retina.

J. A. Martin-Pereda (S'63-M'70-SM'91) received the Ph.D. degree from E.T.S. Ingenieros de Telecomuncación, Universidad Politécnica de Madrid (UPM), Madrid, Spain, in 1971.

He has been a full Professor with UPM since 1975 and was a member of Several Technical Programme Conferences, mainly in Optical Communications (ECOC, OFC, IOOC) from 1985 until 1994. He has published more than 100 papers in journals and books and was the recipient of several academic and technical prizes. His present interest is the study of biological systems on models for artificial systems.

Prof. Martin-Pereda is a member of the Royal Spanish Academy of Engineering, the Optical Society of America, and SPIE. 